NASA/TM-2015-218737

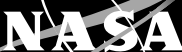

Tunable Magnetic Resonance in Microwave Spintronics Devices

Yunpeng Chen

University of Delaware, Newark, Delaware

Xin Fan

University of Denver, Denver, Colorado

Yunsong Xie, Yang Zhou, and Tao Wang

University of Delaware, Newark, Delaware

Jeffrey D. Wilson and Rainee N. Simons

Glenn Research Center, Cleveland, Ohio

Sui-Tat Chui and John Q. Xiao

University of Delaware, Newark, Delaware 


\section{NASA STI Program . . . in Profile}

Since its founding, NASA has been dedicated to the advancement of aeronautics and space science. The NASA Scientific and Technical Information (STI) Program plays a key part in helping NASA maintain this important role.

The NASA STI Program operates under the auspices of the Agency Chief Information Officer. It collects, organizes, provides for archiving, and disseminates NASA's STI. The NASA STI Program provides access to the NASA Technical Report Server-Registered (NTRS Reg) and NASA Technical Report ServerPublic (NTRS) thus providing one of the largest collections of aeronautical and space science STI in the world. Results are published in both non-NASA channels and by NASA in the NASA STI Report Series, which includes the following report types:

- TECHNICAL PUBLICATION. Reports of completed research or a major significant phase of research that present the results of NASA programs and include extensive data or theoretical analysis. Includes compilations of significant scientific and technical data and information deemed to be of continuing reference value. NASA counter-part of peer-reviewed formal professional papers, but has less stringent limitations on manuscript length and extent of graphic presentations.

- TECHNICAL MEMORANDUM. Scientific and technical findings that are preliminary or of specialized interest, e.g., "quick-release" reports, working papers, and bibliographies that contain minimal annotation. Does not contain extensive analysis.
- CONTRACTOR REPORT. Scientific and technical findings by NASA-sponsored contractors and grantees.

- CONFERENCE PUBLICATION. Collected papers from scientific and technical conferences, symposia, seminars, or other meetings sponsored or co-sponsored by NASA.

- SPECIAL PUBLICATION. Scientific, technical, or historical information from NASA programs, projects, and missions, often concerned with subjects having substantial public interest.

- TECHNICAL TRANSLATION. Englishlanguage translations of foreign scientific and technical material pertinent to NASA's mission.

For more information about the NASA STI program, see the following:

- Access the NASA STI program home page at http://www.sti.nasa.gov

- E-mail your question to help@sti.nasa.gov

- Fax your question to the NASA STI Information Desk at 757-864-6500

- Telephone the NASA STI Information Desk at 757-864-9658

- Write to:

NASA STI Program

Mail Stop 148

NASA Langley Research Center Hampton, VA 23681-2199 
NASA/TM-2015-218737

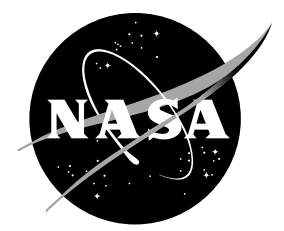

\section{Tunable Magnetic Resonance in Microwave Spintronics Devices}

Yunpeng Chen

University of Delaware, Newark, Delaware

Xin Fan

University of Denver, Denver, Colorado

Yunsong Xie, Yang Zhou, and Tao Wang

University of Delaware, Newark, Delaware

Jeffrey D. Wilson and Rainee N. Simons

Glenn Research Center, Cleveland, Ohio

Sui-Tat Chui and John Q. Xiao

University of Delaware, Newark, Delaware

Prepared for the

2015 International Microwave Symposium

sponsored by the Institute of Electrical and Electronics Engineers

Phoenix, Arizona, May 17-22, 2015

National Aeronautics and

Space Administration

Glenn Research Center

Cleveland, Ohio 44135 


\section{Acknowledgments}

The University of Delaware authors acknowledge the support that they have received from the NASA EPSCoR Program under contract number NNX11AQ29A.

\section{Supplementary Notes}

The figures are archived under E-19066.

This report contains preliminary findings, subject to revision as analysis proceeds.

Level of Review: This material has been technically reviewed by technical management.

Available from

NASA STI Program

Mail Stop 148

NASA Langley Research Center

Hampton, VA 23681-2199
National Technical Information Service 5285 Port Royal Road Springfield, VA 22161

703-605-6000

This report is available in electronic form at http://www.sti.nasa.gov/ and http://ntrs.nasa.gov/ 


\title{
Tunable Magnetic Resonance in Microwave Spintronics Devices
}

\author{
Yunpeng Chen \\ University of Delaware \\ Newark, Delaware 19716 \\ Xin Fan \\ University of Denver \\ Denver, Colorado 80208 \\ Yunsong Xie, Yang Zhou, and Tao Wang \\ University of Delaware \\ Newark, Delaware 19716 \\ Jeffrey D. Wilson and Rainee N. Simons \\ National Aeronautics and Space Administration \\ Glenn Research Center \\ Cleveland, Ohio 44135 \\ Sui-Tat Chui and John Q. Xiao \\ University of Delaware \\ Newark, Delaware 19716
}

\begin{abstract}
Magnetic resonance is one of the key properties of magnetic materials for the application of microwave spintronics devices. The conventional method for tuning magnetic resonance is to use an electromagnet, which provides very limited tuning range. Hence, the quest for enhancing the magnetic resonance tuning range without using an electromagnet has attracted tremendous attention. In this paper, we exploit the huge exchange coupling field between magnetic interlayers, which is on the order of $4000 \mathrm{Oe}$ and also the high frequency modes of coupled oscillators to enhance the tuning range. Furthermore, we demonstrate a new scheme to control the magnetic resonance frequency. Moreover, we report a shift in the magnetic resonance frequency as high as $20 \mathrm{GHz}$ in CoFebased tunable microwave spintronics devices, which is $10 \mathrm{X}$ higher than conventional methods.
\end{abstract}

\subsection{Introduction}

Spintronics utilizes electron spins in addition to, and sometimes in place of, electron charges to achieve new electron device functions. The discovery of giant magnetoresistance (GMR) in 1988 revolutionized the data storage in hard drives (Ref. 1). More recently, the discovery of tunnel magnetoresistance (TMR) phenomena in magnetic tunnel junctions (MTJs) accelerated the development of hard drive technology. Besides reading out the static magnetic moment (stored information) of a nano-magnet in, for example, a computer hard disk, the development of spintronics also offers a new scheme to manipulate the magnetic moment by means of spin transfer torque (STT). The STT transfers angular momentum from spin- polarized charge carriers to local magnetic moments without using an electromagnet. Owing to its fast speed, high density, and low energy consumption, STT has been utilized in developing the next generation of magnetic random access memories (MRAM). Another emerging subfield of spintronics involves coupling spin transport to spin dynamics. Since spin dynamics usually occurs in the gigahertz frequency range due to the intrinsic resonance of magnetic materials, spintronics devices have found applications at microwave frequencies, for example in microwave generators, detectors, and phase shifters (Refs. 2 to 4 ).

The typical spintronic devices include spin valves (SVs) and MTJs, which are respectively, made of a nonmagnetic metal or insulating barrier sandwiched between two ferromagnetic (FM) layers as illustrated in Figure 1(a). One of the FM layers usually has its magnetic moment $\left(M_{\text {fixed }}\right)$ fixed and aligned with the adjacent antiferromagnetic (AFM) layer through exchange bias. The other layer's magnetic moment $\left(M_{\text {free }}\right)$ is free to rotate with the magnetic fields. The electric resistance of the structure is determined by the relative angle between $M_{\text {fixed }}$ and $M_{\text {free }}$. When a current is flowing through the stack, the electron spins are polarized along the direction of $M_{\text {fixed }}$ (Fig. 1(a)). If $M_{\text {free }}$ is not collinear with $M_{\text {fixed }}$, the electrons with polarized spins passing through the free layer will align with $M_{\text {free }}$ due to exchange interaction. By the conservation of angular momentum, the change of the carrier spin momentum will transfer to $M_{\text {free }}$, which is the spin transfer torque.

To generate the spin current without passing a charge current through a magnetic layer, one can pass a spin current through a material with strong spin orbital coupling (SOC) such as heavy metals (HM) like Pt or topological insulators (Ref. 5). Due to SOC, the electrons with opposite spins will be separated in the 
(a)
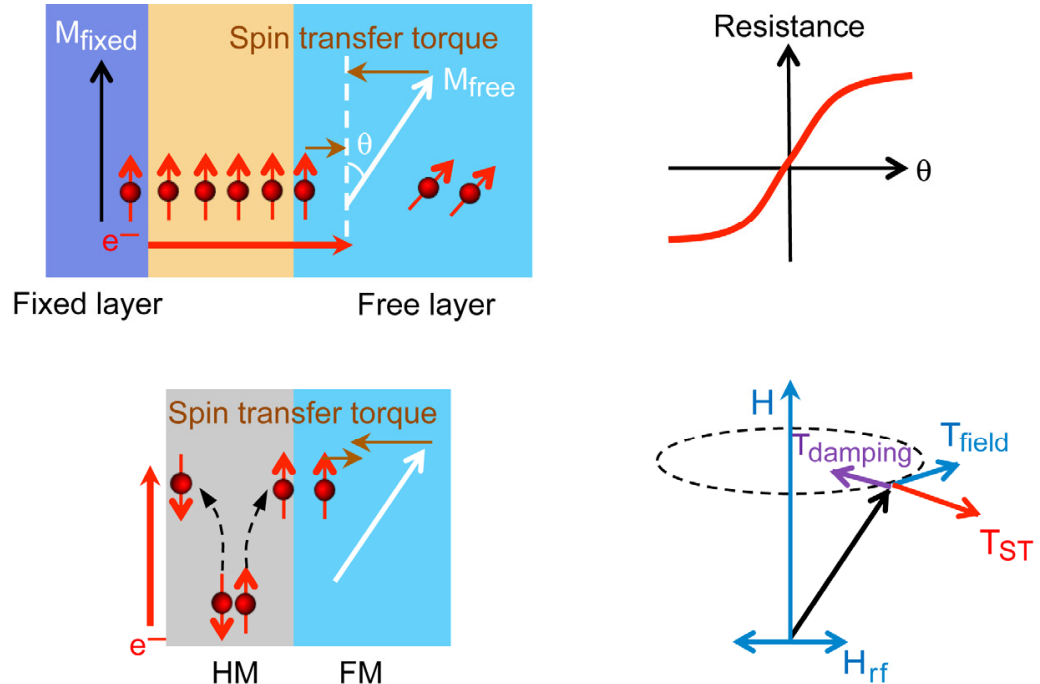

(b)
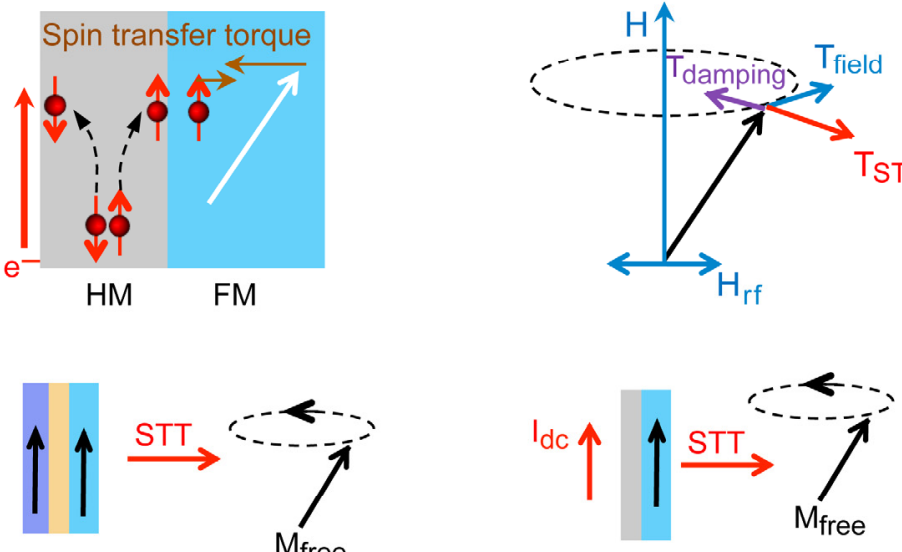

(c)

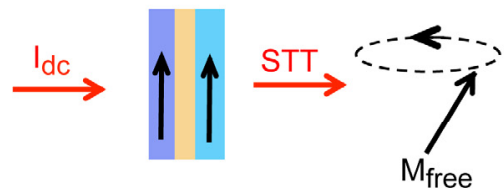

(d)

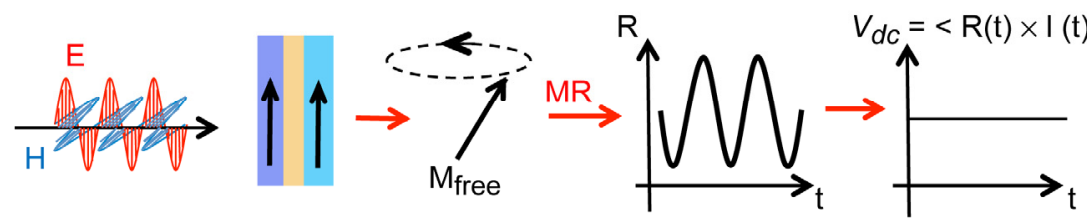

(e)

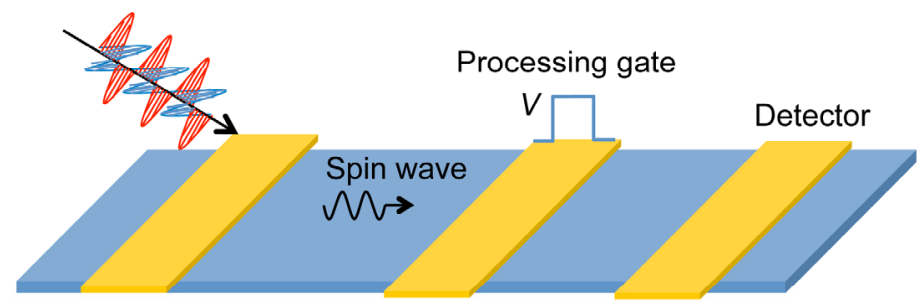

Figure 1.-(a) Spin transfer torque in spintronics device and the magnetoresistance as a function of the relative angle between the magnetization directions in two FM layers. (b) Spin transfer torque due to the spin Hall effect (SHE) and the torque on local magnetic moment. (c) Spin-torque microwave oscillator where microwaves are generated by passing a dc current through the spintronic device. (d) Spintronics microwave detector. (e) Spin wave logic device.

direction transverse to the electric current (Fig. 1(b)), which is known as the spin Hall effect (SHE). The transverse spin current diffuses into the adjacent FM layer and manipulates its magnetic configuration as illustrated in Figure 1(b). The STT $\left(T_{S T}\right)$ is aligned with the damping torque ( $\left.\mathrm{T}_{\text {damping }}\right)$. Depending on the sign of the injected spin, the STT will either enhance the damping or act as an anti-damping torque. STT is able to reduce the device noise by compensating the damping, exciting magnetic resonance and emitting microwaves by sustaining magnetization precession. Figure 1(c) illustrates the principle of a nano-STT-microwave oscillator. The spintronics devices can also work as nano-microwave detectors based on the spin rectification effect. As depicted in Figure 1(d), the magnetization precession is excited through the interaction of microwave magnetic or electric fields. In a SV or an MTJ, the rotation of free layer magnetization will cause a time variant resistance. The ac resistance rectifies the microwave field, resulting in a dc voltage output. Moreover, the microwave field can be used to excite a spin wave (the wave of angular momentum oscillation) in a spin-wave logic device with low operating energy (Fig. 1(e)), which has the potential to be more energy efficient than the conventional complementary metal oxide semiconductor (CMOS) technology. 
In conventional tunable microwave spintronic devices, the magnetic resonance is controlled by an external applied magnetic field, which is generated by a bulky and energy inefficient electromagnet. To integrate these devices and to lower the power consumption, it is desirable to eliminate the use of an electromagnet. Researchers have demonstrated that the magnetic resonance in piezoelectric/ferromagnetic multiferroic heterostructures can be controlled by an external electric field. However, no material satisfies both tunable range and microwave performance simultaneously. This paper reports a new technique to control the magnetic resonance, which is demonstrated by exploiting the huge interlayer exchange coupling field present between the device magnetic layers and the high frequency modes of coupled oscillators.

\subsection{Magnetic Resonance Modes in Exchange Coupled Magnetic Bilayer}

Exchange interaction exists inside every magnetic material and also at the interface of two different magnetic materials. In a typical ferromagnetic material, the exchange field arising from the Pauli Exclusion Principle can be as high as $10^{3} \mathrm{~T}$, equivalent to a $28 \mathrm{THz}$ frequency range. The idea of using two coupled layers FM1 and FM2 resembles two coupled oscillators, which exhibit low frequency acoustic and high frequency optical modes, and the sensitivity of the latter will depend on the coupling at the interface. We model the interface arising from the dominating exchange coupling between two nearest neighboring layers and neglect the much smaller dipolar interaction. The interlayer exchange energy per unit area is described as

$$
E=-J \frac{\vec{M}_{1} \cdot \vec{M}_{2}}{M_{s 1} \cdot M_{s 2}}
$$

where $\vec{M}_{1}$ and $\vec{M}_{2}$ are the magnetization vectors of FM1 and FM2, $M_{s 1}$ and $M_{s 2}$ are the saturation magnetizations of FM1 and FM2, respectively, and $J$ is the interface exchange coupling strength. The coupled magnetization dynamics can then be described by the modified Landau-Lifshitz-Gilbert (LLG) equation as

$$
\frac{d \vec{M}_{i}}{d t}=-\gamma \mu_{0} \vec{M}_{i} \times\left(\vec{H}_{e f f_{-} i}+\vec{H}_{e_{-} i}\right)+\frac{\alpha_{i}}{M_{s_{-} i}} \vec{M}_{i} \times \frac{d \vec{M}_{i}}{d t}
$$

where $\gamma$ is the gyromagnetic ratio of $28 \mathrm{GHz} / \mathrm{T}, \vec{H}_{\text {eff }} i$ is the effective field due to other atomic layers on the $i$-th layer including the external field, anisotropy field and demagnetizing field, $\vec{H}_{e_{-} i}$ is the effective exchange field on the $i$-th layer, $\alpha_{i}$ is the damping of the $i$-th layer, and $M_{s_{-} i}$ is the saturation magnetization of the $i$-th layer.

We are able to simulate the permeability spectrum of the exchange coupled layer. Indeed, when the interface exchange coupling is finite but much weaker than the intralayer exchange coupling inside each ferromagnetic layer, the bilayer exhibits two microwave-resonance modes as illustrated in Figure 2. The two modes are the acoustic mode with the magnetizations in two layers precessing in-phase and the optical mode with the two magnetizations precessing out-of-phase. In a simplified example, where FM1 and FM2 are identical with negligible anisotropy, i.e., $M_{s 1}=M_{s 2}=M_{s}$ and $d_{1}=d_{2}=d$, the two resonance modes can be calculated as

$$
\omega_{\text {acoustic }}=\gamma \mu_{0} \sqrt{H_{e f f}\left(H_{e f f}+M_{s}\right)} \text { and }
$$

$$
\omega_{\text {optical }}=\gamma \mu_{0} \sqrt{\left(\frac{2 J}{\mu_{0} M_{s} d}+H_{e f f}\right)\left(\frac{2 J}{\mu_{0} M_{s} d}+H_{e f f}+M_{s}\right)}
$$

In the above equations $H_{\text {eff }}$ is the effective magnetic field. The optical mode has a higher resonance frequency than the acoustic mode from the contribution of an additional effective exchange field $2 J / \mu_{0} M_{s} d$. The resonance frequency of the optical mode can be shifted by the change of the interlayer exchange coupling.
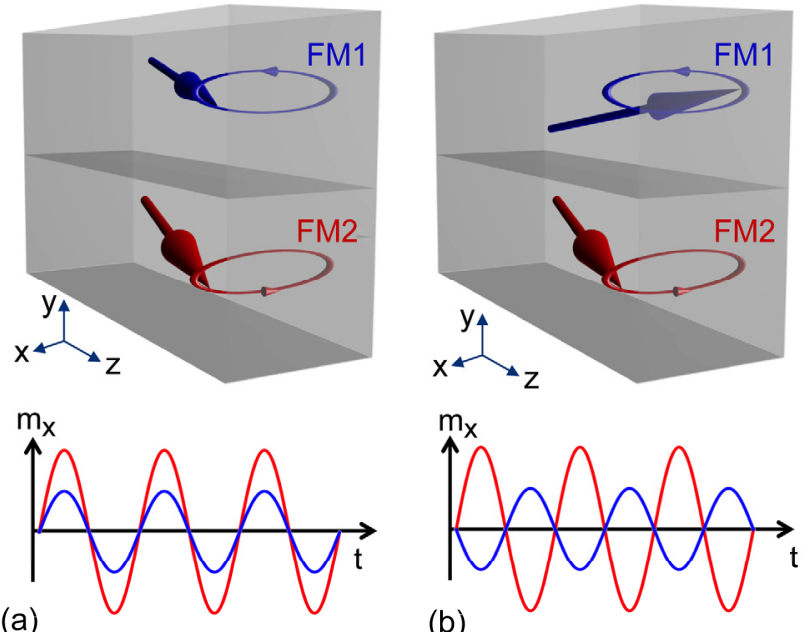

(b)

Figure 2.-Two microwave resonance modes: (a) acoustic and (b) optical mode. The bottom graphs show the in-phase magnetization oscillations for the acoustic-mode and out-of-phase magnetization oscillations for the optical mode. 


\subsection{Electric Field Tuned Magnetic Resonance in Multiferroic Heterostructures}

The application of an electric field induces strain in piezoelectric materials. The strain-induced magnetoelectric (ME) interaction was recently demonstrated to be a practical method to tune the magnetic resonance in layered multiferroic heterostructures (Refs. 8 to 14). A comparison of electric field induced resonant field shift in various multiferroic heterostructures is summarized in Table I. A typical experimental configuration is illustrated in Figure 3(a). FM layers were deposited on a piezoelectric substrate, such as $\mathrm{Pb}\left(\mathrm{Mg}_{1 / 3} \mathrm{Nb}_{2 / 3}\right) \mathrm{O}_{3}-\mathrm{PbTiO}_{3}(\mathrm{PMN}-\mathrm{PT})$ and $\mathrm{Pb}\left(\mathrm{Zn}_{1 / 3} \mathrm{Nb}_{2 / 3}\right) \mathrm{O}_{3}-$ $\mathrm{PbTiO}_{3}$ (PZN-PT). The magnetic resonance was detected by microwave transmission. In the presence of an electric field, the piezoelectric substrate will strain the FM layers, which introduces an effective anisotropy field due to the magnetostrictive effect. The largest strain-induced effective magnetic field of 3500 Oe was achieved in terfenol-D/PZN-PT (Ref. 6) because of the high magnetostriction of terfenol-D. However, the ferromagnetic resonance (FMR) linewidth was also large indicating enormous loss. Besides the magnetrostrictive effect, the change of interlayer exchange coupling may also contribute to the shift of resonance frequency, because the exchange coupling will be affected by the strain. We deposited $\mathrm{Co}_{10} \mathrm{Fe}_{90}(40 \mathrm{~nm}) / \mathrm{Ni}_{80} \mathrm{Fe}_{20}(40 \mathrm{~nm})$ on PZNPT(011). We observed an effective magnetic field of 820 Oe when we applied a bias electric field as shown in Figure 3(b) and (c). The effective magnetic field is also known as the tunable magnetic field or the electric field induced magnetic anisotropy field $(\Delta \mathrm{H})$. The control sample with a single $\mathrm{CoFe}$ layer on PZN-PT shows a smaller effective magnetic field of 600 Oe. The 220 Oe difference is due to the change of interlayer exchange coupling. Therefore, the interlayer exchange coupling is not efficiently tuned by strain. A much more effective controlling method is demonstrated in the next section.
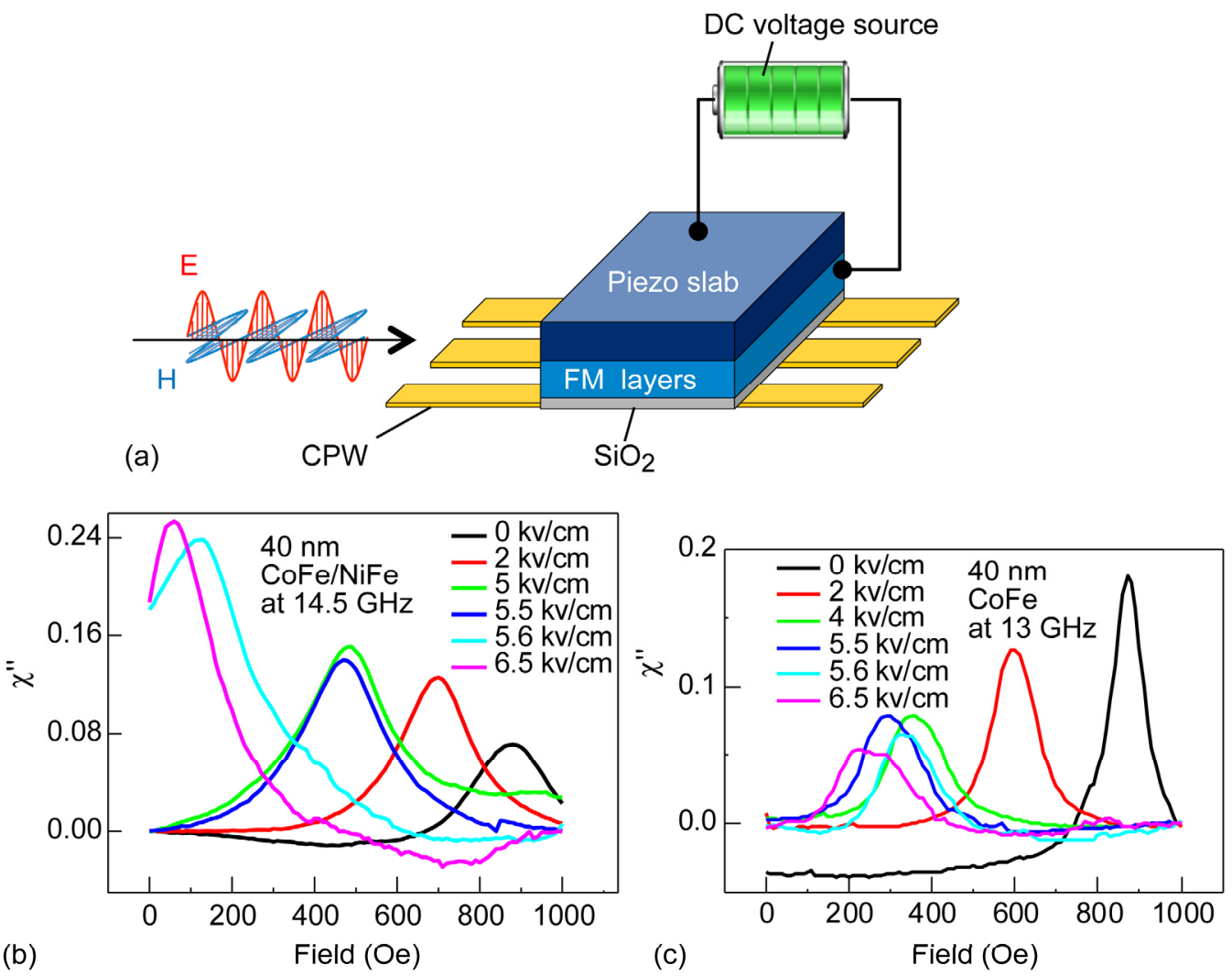

Figure 3.-(a) Measurement setup for the electric field controlled magnetic resonance frequency. Measured susceptibility as a function of the external applied magnetic field with bias electric field as a parameter for (b) $\mathrm{Co}_{10} \mathrm{Fe} 90(40 \mathrm{~nm}) / \mathrm{Ni80Fe} 20(40 \mathrm{~nm})$ structure at $14.5 \mathrm{GHz}$ and (c) $\mathrm{Co}_{10} \mathrm{Fe}_{90}(40 \mathrm{~nm})$ structure at $13.0 \mathrm{GHz}$. 


\subsection{Giant Resonance Frequency Tunability Using Exchange Coupling}

To efficiently tune the resonance frequency of the optical mode, one can adjust the interlayer exchange coupling $J$ by engineering the interface. A tantalum (Ta) spacer layer was deposited in between two FM layers. The interlayer exchange coupling can be adjusted by the thickness of the Ta layer. Although the magnetization precessions of the two magnetic layers are out-of-phase at the optical mode, it is still suitable for the application of spintronics microwave devices, because the ferromagnetic layer near the nonmagnetic/insulator interface governs the performance of the devices (Ref. 9). We use a spin valve based microwave detector to demonstrate the effect of the optical mode on microwave detection. As shown in Figure 4(a), the detector is a strip of spin valve (SV), in which the free magnetic layer is composed of an exchange coupled ferromagnetic bilayer with a discontinuous nonmagnetic layer of varying thickness in between to tune the strength of exchange coupling, $\mathrm{Cu}(2 \mathrm{~nm}) / \mathrm{Co}_{10} \mathrm{Fe}_{90}(4 \mathrm{~nm}) / \mathrm{Ta}(0.4-2.0 \mathrm{~nm}) / \mathrm{Ni}_{80} \mathrm{Fe}_{20}$ $(4 \mathrm{~nm}) / \mathrm{Cu}(3 \mathrm{~nm}) / \mathrm{Co}_{10} \mathrm{Fe}_{90}(4 \mathrm{~nm}) / \mathrm{IrMn}(15 \mathrm{~nm}) / \mathrm{Ta}(5 \mathrm{~nm})$. The SV is patterned as a part of a coplanar waveguide (CPW), which guides microwave fields into the SV detector. The microwave magnetic field excites the acoustic mode as well as the optical mode in the bilayer. The precession of the magnetization in the bilayer results in an SV with ac resistance that rectifies the microwave field and generates a dc voltage analogous to the spin-torque diode microwave detectors (Ref. 7). In this case, only the NiFe layer that is adjacent to the $3 \mathrm{~nm} \mathrm{Cu}$ layer contributes to the resistance change in the SV. The dc voltage is proportional to the microwave power and attains a maximum value at the acoustic mode and the optical mode resonances.

The measured voltage peaks corresponding to the acoustic mode and the optical mode with opposite phase are shown in Figure 4(b). In this figure the red arrows indicate the optical mode and the blue arrows indicate the acoustic mode. By varying the thickness of the discontinuous Ta spacer layer, the resonance frequency due to the optical mode can be shifted

(a)

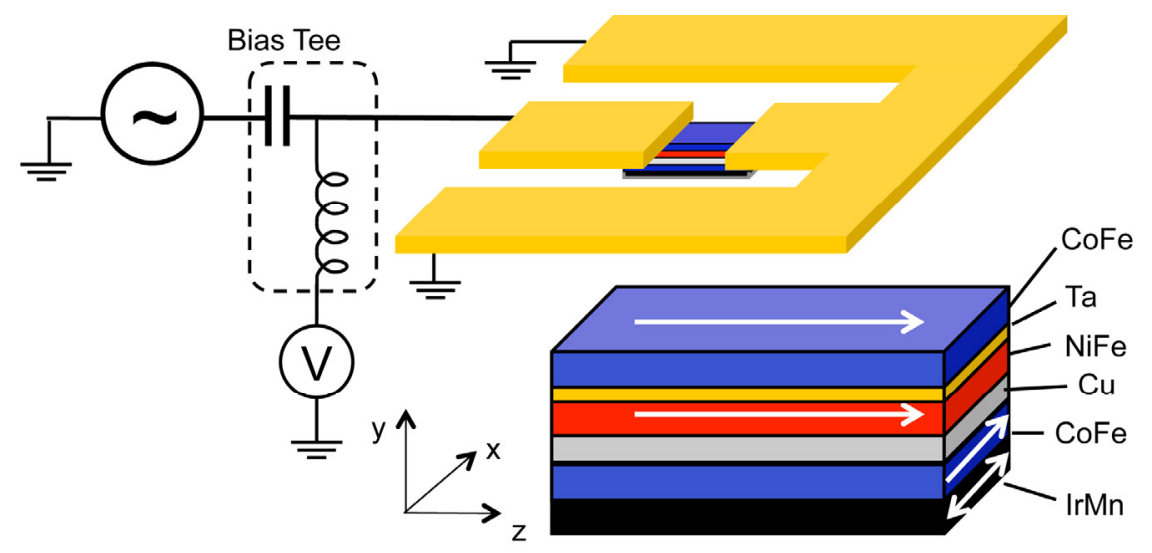

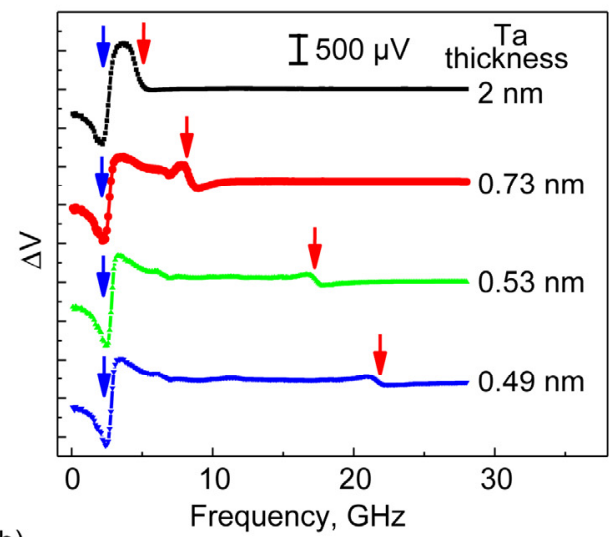

(b)

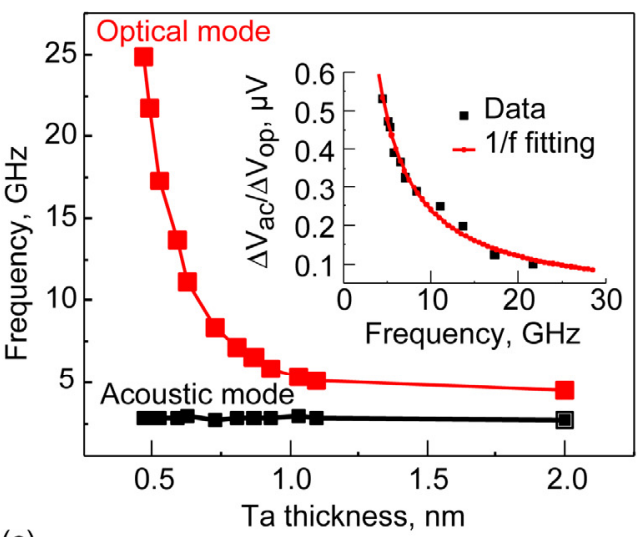

(c)

Figure 4.-(a) Measurement setup of the spin valve-based microwave detector. (b) Measured rectified voltage as a function of frequency with Ta layer thickness as a parameter. (c) Measured acoustic-mode and optical-mode resonance frequencies as a function of Ta layer thickness. The inset shows that voltage sensitivity is inversely proportional to the resonance frequency. The acoustic mode is used here to calibrate the input microwave power. 
TABLE I.-COMPARISON OF ELECTRIC FIELD INDUCED CHANGE OF THE MAGNETIC ANISOTROPY FIELD $(\Delta H)$ AND MAGNETOELECTRIC COUPLING COEFFICIENT $(\Delta H / \Delta E)$ IN VARIOUS MULTIFERROIC HETEROSTRUCTURES

\begin{tabular}{|l|c|c|c|c|}
\hline \multicolumn{1}{|c|}{ Structure } & $\begin{array}{c}\Delta H \\
(\mathrm{Oe})\end{array}$ & $\begin{array}{c}\Delta H / \Delta E \\
\left(\mathrm{Oe} \mathrm{cm} \mathrm{kV} \mathrm{kV}^{-1}\right)\end{array}$ & Linewidth & Reference \\
\hline CoFe/NiFe/PZN-PT & 820 & 126 & 170 Oe at $14.5 \mathrm{GHz}$ & This study \\
\hline CoFe/PZN-PT & 600 & 96 & 90 Oe at $13 \mathrm{GHz}$ & This study \\
\hline Terfenol-D/PZN-PT & 3500 & 580 & 1200 Oe at $9.3 \mathrm{GHz}$ & $(6)$ \\
\hline NiFe/PMN-PT & 375 & 60 & 50 Oe at $9.5 \mathrm{GHz}$ & $(9)$ \\
\hline FeCoHf/PZN-PT & 500 & 82 & 25 Oe at $11.85 \mathrm{GHz}$ & $(10)$ \\
\hline FeGaB/PMN-PT & 330 & 33 & 50 Oe at $9.5 \mathrm{GHz}$ & $(11)$ \\
\hline FeGaB/PZN-PT & 750 & 94 & 50 Oe at $9.6 \mathrm{GHz}$ & $(12)$ \\
\hline FeO/PZN-PT & 600 & 100 & 400 Oe at $9.3 \mathrm{GHz}$ & $(13)$ \\
\hline YIG/PMN-PT & 40 & 5.4 & 6 Oe at $9.3 \mathrm{GHz}$ & $(14)$ \\
\hline
\end{tabular}

from 5 to $25 \mathrm{GHz}$ as shown in Figure 4(c). The corresponding effective field change is $4000 \mathrm{Oe}$, which is much larger than the strain-induced effective field listed in Table I. The magnitude of the resonance peaks scale inversely with the resonance frequency following the nature of magnetic resonance, as shown in the inset of Figure 4(c). For in-situ tuning of interlayer exchange coupling, one can replace the Ta spacer layer with a phase transition material, such as $\mathrm{NiCu}$ alloy, which is sensitive to temperature or mechanical strain (Ref. 8).

\subsection{Conclusion}

We have demonstrated a technique to significantly enhance the ferromagnetic resonance frequency by evoking the exchange mode in magnetic bilayers. The ferromagnetic exchange coupling in magnetic bilayers enables an extremely wide frequency tuning range. We report a technique to shift the magnetic resonance frequency as high as $20 \mathrm{GHz}$ in CoFe-based microwave spintronic devices, which is $10 \mathrm{X}$ higher than conventional techniques (Ref. 9). Such a wide tuning range is normally not achievable with applying an external magnetic field or by other known tuning methods. Although the demonstrations are done with thin film structures, which are already applicable to a plethora of spintronics devices, the concept can be extended to thick materials with multiple layers, creating new avenues for magnetic materials based microwave devices.

\section{References}

1. M. N. Baibich, J. M. Broto, A. Fert, F. N. Vandau, F. Petroff, P. Eitenne, G. Creuzet, A. Friederich, and J. Chazelas, "Giant magnetoresistance of $(001) \mathrm{Fe} /(001) \mathrm{Cr}$ magnetic superlattices," Physical Review Letters, vol. 61, no. 21, pp. 2472-2475, Nov 21, 1988.

2. D. Houssameddine, S. H. Florez, J. A. Katine, J. P. Michel, U. Ebels, D. Mauri, O. Ozatay, B. Delaet, B. Viala, L. Folks, B. D. Terris, and M. C. Cyrille, "Spin transfer induced coherent microwave emission with large power from nanoscale $\mathrm{MgO}$ tunnel junctions," Appl. Phys. Lett., vol. 93, no. 2, 14 July 2008, 022505 (2008); http://dx.doi.org/10.1063/1.2956418.
3. X. Fan, R. Cao, T. Moriyama, W. Wang, H. W. Zhang, and J. Q. Xiao, "Magnetic tunnel junction based microwave detector," Appl. Phys. Lett., vol. 95, no.12, 21 Sept 2009, 122501 (2009); http://dx.doi.org/10.1063/ 1.3231874 .

4. C. S. Tsai and J. Su, "A wideband electronically tunable microwave notch filter in yttrium iron garnet-gallium arsenide material structure," Appl. Phys. Lett., vol. 74, no. 14, 5 April 1999, 2079 (1999); http://dx.doi.org/10.1063/ 1.123763.

5. X. Fan, J. Wu, Y. Chen, M. J. Jerry, H. Zhang, and J. Q. Xiao, "Observation of the nonlocal spin-orbital effective field," Nature Communications, vol. 4, Article number: 1799, 30 April 2013; DOI: 10.1038/ncomms2709.

6. M. Liu, S. Li, Z. Zhou, S. Beguhn, J. Lou, F. Xu, T. J. Lu, and N. X. Sun, "Electrically induced enormous magnetic anisotropy in Terfenol-D/lead zinc niobate-lead titanate multiferroic heterostructures," J. Appl. Phys. vol. 112, no. 6, 15 Sept 2012, 063917 (2012); http://dx.doi.org/ $10.1063 / 1.4754424$.

7. A. A. Tulapurkar, Y. Suzuki, A. Fukushima, H. Kubota, H. Maehara, K. Tsunekawa, D. D. Djayaprawira, N. Watanabe, and S. Yuasa, "Spin-torque diode effect in magnetic tunnel junctions," Nature, vol. 438, pp. 339-342, 17 Nov 2005; DOI: 10.1038/nature04207.

8. Y. Chen, X. Fan, Y. Zhou, Y. Xie, J. Wu, T. Wang, S. T. Chui, and J. Q. Xiao, "Designing and tuning magnetic resonance with exchange interaction," Advanced Materials, vol. 27, no. 8, pp. 1351-1355, 25 Feb 2015; DOI: 10.1002/adma.201404447.

9. T. Nan, Z. Zhou, M. Liu, X. Yang, Y. Gao, B. A. Assaf, H. Lin, S. Velu, X. Wang, H. Luo, J. Chen, S. Akhtar, E. Hu, R. Rajiv, K. Krishnan, S. Sreedhar, D. Heiman, B. M. Howe, G. J. Brown, and N. X. Sun, "Quantification of strain and charge co-mediated magnetoelectric coupling on ultra-thin Permalloy/PMN-PT interface," Scientific Reports, vol. 4, Article number: 3688, 14 Jan 2014; DOI: 10.1038/srep03688. 
10. S. Li, H. Du, Q. Xue, X. Gao, Y. Zhang, W. Shao, T. Nan, Z. Zhou, and N. X. Sun, "Large E-field tunability of microwave ferromagnetic properties in $\mathrm{Fe}_{59.3} \mathrm{Co}_{28.0} \mathrm{Hf}_{12.7} /$ PZN-PT multiferroic composites," J. Appl. Phys. vol. 115, no. 17, 7 May 2014, 17C723 (2014); http://dx.doi.org/10.1063/1.4865315

11. L. Jing, D. Reed, M. Liu, C. Pettiford, and N. X. Sun, "Novel electrostatically tunable FeGaB/(Si)/PMN-PT multiferroic heterostructures for microwave application," 2009 IEEE MTT-S International Microwave Symposium Digest, pp. 33-36, Boston, MA, 7-12 June 2009; DOI: 10.1109/MWSYM.2009.5165625

12. J. Lou, M. Liu, D. Reed, Y. Ren, and N. X. Sun, "Giant electric field tuning of magnetism in novel Multiferroic FeGaB/Lead Zinc Niobate-Lead titanate (PZN-PT) heterostructures," Advanced Materials, vol. 21, no. 46, pp. 4711-4715, 11 Dec 2009; DOI: 10.1002/ adma.200901131
13. M. Liu, O. Obi, Z. Cai, J. Lou, G. Yang, K. S. Ziemer, and N. X. Sun, "Electrical tuning of magnetism in $\mathrm{Fe}_{3} \mathrm{O}_{4} / \mathrm{PZN}-\mathrm{PT}$ multiferroic heterostructures derived by reactive magnetron sputtering," J. Appl. Phys. vol. 107, no. 7, 1 April 2010, 073916 (2010); http://dx.doi.org/ 10.1063/1.3354104

14. S. Shastry, G. Srinivasan, M. I. Bichurin, V. M. Petrov, and A. S. Tatarenko, "Microwave magnetoelectric effects in single crystal bilayers of yttrium iron garnet and lead magnesium niobate-lead titanate," Phys. Rev. B, vol. 70, no. 6, 1 Aug 2004, 064416 (2004); http://dx.doi.org/ 10.1103/PhysRevB.70.064416 


